\title{
Farewell to "W" (Herbert Saul Wilf), a True VISIONARY for Whom EVERYTHING was INTERTWINED
}

\author{
Doron Zeilberger
}

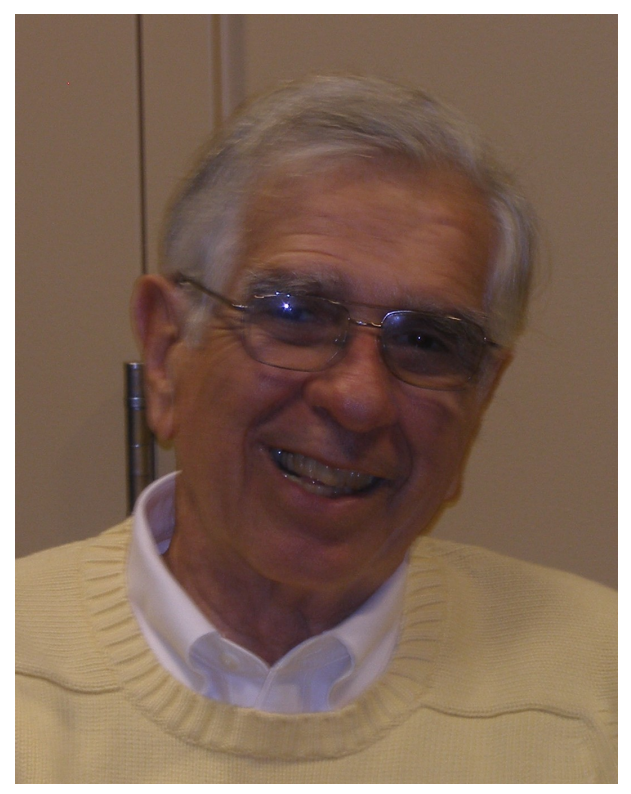

The better half of WZ pair [1], the "W", the first (and the more complex one of the) "pair of complex variables" (see footnote at p. 2 of the masterpiece [2]) left this world on Jan. 7, 2012. But only his body!

$\mathrm{W}$ was an outstanding combinatorialist, of course, but as I have already said in the tribute [3] his influence and vision far transcends any one specialty.

In the Philadelphia Inquirer obit [4], W's wife of 59 plus year, midwife guru Ruth Tumen Wilf, is quoted saying that "for him, teaching and research were deeply intertwined". True, of course, but not only "teaching and research". Also pure and applied, conceptual and computational, problem solving and theory building, global and local, strategic and tactical and so on and so forth.

It is a mark of an interesting person that his or her children are interesting and diversified in their talents, interests, and activities. Herb had three wonderful children: Susan [5], a brilliant Chinese scholar, David [6], a brilliant lawyer, and Peter [7], a brilliant paleontologist. And he had twenty eight brilliant academic children [8], all different than each other, including a bishop [9]! He also has six brilliant biological grandchildren, and 21 talented academic grandchildren.

And Herb was such a mensch. He made sure that Sister Celine [10] would get the recognition that she so deserved, and flew his plane to her convent, in order to conduct a historic interview [11] that is preserved for posterity.

$\mathrm{W}$ was also one of the greatest lecturers that I have ever known. For an example, watch his great lecture whimsically titled "How to lose as little as possible": [12]

And $\mathrm{W}$ was not only a great problem-solver, but like Erdős, also a great problem-poser. Please try to solve any of his unsolved problems [13], that he only posted a year ago. Myself, I have no clue how to solve 
any of them, and I could only say something [14] about the sixth problem.

On Feb. 2, 2012, I gave a talk about W at the Rutgers Experimental Mathematics Seminar [15], that was (like all talks in that seminar where the speaker agrees) videotaped and uploaded to YouTube [16]. There I highlighted some of my favorite W gems, including the Greene-Nijenhuis-Wilf probabilistic proof of the hook-length-formula [17] and the gorgeous Calkin-Wilf Recounting of Fractions [18].

\section{References}

[1] http://en.wikipedia.org/wiki/Wilf\%E2\%80\%93Zeilberger_pair

[2] http://www.math.rutgers.edu/\%7Ezeilberg/mamarim/mamarimPDF/rational.pdf

[3] http://www .math.rutgers.edu/\%7Ezeilberg/mamarim/mamarimPDF/w80.pdf

[4] http://articles.philly.com/2012-01-15/news/30629212_1_american-mathematical-society -penn-faculty-assistant-professor

[5] http://paper-republic.org/translators/susan-wilf/

[6] http://www.gibsondunn.com/lawyers/dwilf

[7] http://www3.geosc.psu.edu/\%7Epdw3/

[8] http://www.genealogy.ams.org/id.php?id=15200

[9] http://www.centraldiocesepncc.org/OurBishop.dsp

[10] http://en.wikipedia.org/wiki/Mary_Celine_Fasenmyer

[11] http://vimeo.com/5381704

[12] http://www . youtube.com/watch?v=X4zdU5AHkGM (part 1), http://www . youtube.com/watch?v=EV6buzdX5jo (part 2), http://www . youtube. com/watch?v=w4rf20dK47c (part 3), http://www . youtube. com/watch?v=WSSjwJS3JBE (part 4).

[13] http://www.math.upenn.edu/\%7Ewilf/website/UnsolvedProblems.pdf

[14] http://www .math.rutgers .edu/\%7Ezeilberg/mamarim/mamarimhtml/dmp.html

[15] http://www . math.rutgers .edu/\%7Ebnaka/expmath/

[16] http://www . youtube.com/watch?v=rnFWmswEFFA (part 1), http://www . youtube. com/watch?v=8NwFQGO3tzU (part 2).

[17] http://www . math. upenn.edu/\%7Ewilf/website/Probabilistic\%20proof .pdf

[18] http://www.math. upenn.edu/\%7Ewilf/website/recounting.pdf 